\title{
Coronary stenting using a 6 French diagnostic catheter: A case report
}

\author{
Laxman Dubey,* Sogunuru Guruprasad, Gangapatnam Subramanyama \\ ${ }^{a}$ Department of cardiology, College of Medical sciences and Teaching Hospital, Bharatpur, Nepal
}

\begin{abstract}
Accepted on
June 29th, 2013

DOI Name

http://dx.doi.org/10.3126/jaim.v2i2.8781

\section{Keywords}

diagnostic catheter; feasibility; guiding catheter; percutaneous coronary intervention

\section{Citation}

Laxman Dubey, Sogunuru Guruprasad, Gangapatnam Subramanyam. Coronary stenting using a 6 French diagnostic catheter: A case report. Journal of Advances in Internal Medicine 2013;02(02):71-3.
\end{abstract}

\begin{abstract}
Guiding catheters have larger lumen diameter as compared to the same size diagnostic catheters, and thus provide adequate arterial blood flow and good visualization of the artery during percutaneous coronary intervention. However, feasibility of percutaneous coronary intervention via diagnostic catheters has been demonstrated in a few studies. This case reports the use of a 6 French diagnostic catheter for both the coronary angiography as well as coronary stenting in a 52-year-old male patient with critical mid left anterior descending artery stenosis on December, 2012.
\end{abstract}

\section{INTRODUCTION}

To achieve a successful coronary artery stenting guiding catheter with good back-up support is vital. However, sometimes standard guiding catheters may not selectively cannulate the desired coronary ostium despite a successful cannulation with a diagnostic catheter of the same curve. This might be due to the difference in wall structures of the catheters and a shorter tip and lack of tapering tip of the guiding catheter which can sometimes provide better back-up support than the guiding catheter. ${ }^{1}$ Because the internal lumen diameter of contemporary 6 French (F) guiding catheter ( 0.070 inches) is slightly larger than the internal lumen of $6 \mathrm{~F}$ diagnostic catheters (0.057 inches), it makes easier to pass the balloon catheter and stents and also allows adequate arterial blood flow during the procedure and good visualization of the artery during contrast medium injection. In some cases, if the cannulation of desired coronary artery with guiding catheter following diagnostic angiography is difficult and time taking despite the use of the catheter having similar shape, the use of a diagnostic catheter for coronary stenting may be attractive. Over the recent years, due to the improvement of the technology with a marked decrease in the diameter of balloon catheters and stents, coronary stenting is possible even through smaller guiding catheters. ${ }^{1}$

There are a few studies which have demonstrated the feasibility of percutaneous coronary intervention $(\mathrm{PCl})$ via diagnostic catheters..$^{1-4}$ In the present case, $6 \mathrm{~F}$ diagnostic catheter was used for both the coronary angiography as well as coronary stenting in a patient with critical mid left anterior descending artery (LAD) stenosis.

\section{CASE REPORT}

A 52 year-old man presented to emergency department of college of medical sciences and teaching hospital, Bharatpur, Nepal on December, 2012 complaining of acute onset of left sided chest pain of 4 days which increasing in intensity for 3 hours which was squeezing in character and radiating to the left arm. Pain was associated with profuse sweating and 1 episode of vomiting. He had had hypertension for two years, and was a smoker. Family history was unremarkable for any cardiovascular illness. Physical examination was within normal limits except for a soft systolic murmur at apex. Complete blood count and serum biochemistry were within normal limits. His blood pressure was $110 / 70 \mathrm{mmHg}$ and pulse rate of 75 beats per minutes regular. ECG showed sinus rhythm at the rate of 75 beats per minute with ST segment depression and deep T wave inversion in leads V2-V6. CK-MB was $124 \mathrm{U} / \mathrm{L}$ and Tropo-

\footnotetext{
* Corresponding author

Dr. Laxman Dubey

Department of cardiology,

College of Medical sciences and Teaching Hospital,

Bharatpur-10, Nepal

Email:dubeylax@yahoo.com
} 
nin I was positive. A diagnosis of non ST elevation myocardial infarction was made. Since the patient had ongoing chest pain, coronary angiography was planned. Patient received 300mg aspirin, $600 \mathrm{mg}$ clopidogrel and $80 \mathrm{mg}$ atorvastatin per orally and transferred to the catheterization laboratory.

Coronary angiography was done via right femoral approach. Left coronary artery was hooked with 6 French (F) $3.5 \mathrm{~cm}$ curve Judkins left diagnostic catheter (Cordis) which showed critical stenosis at the middle segment of the LAD (Figure 1).

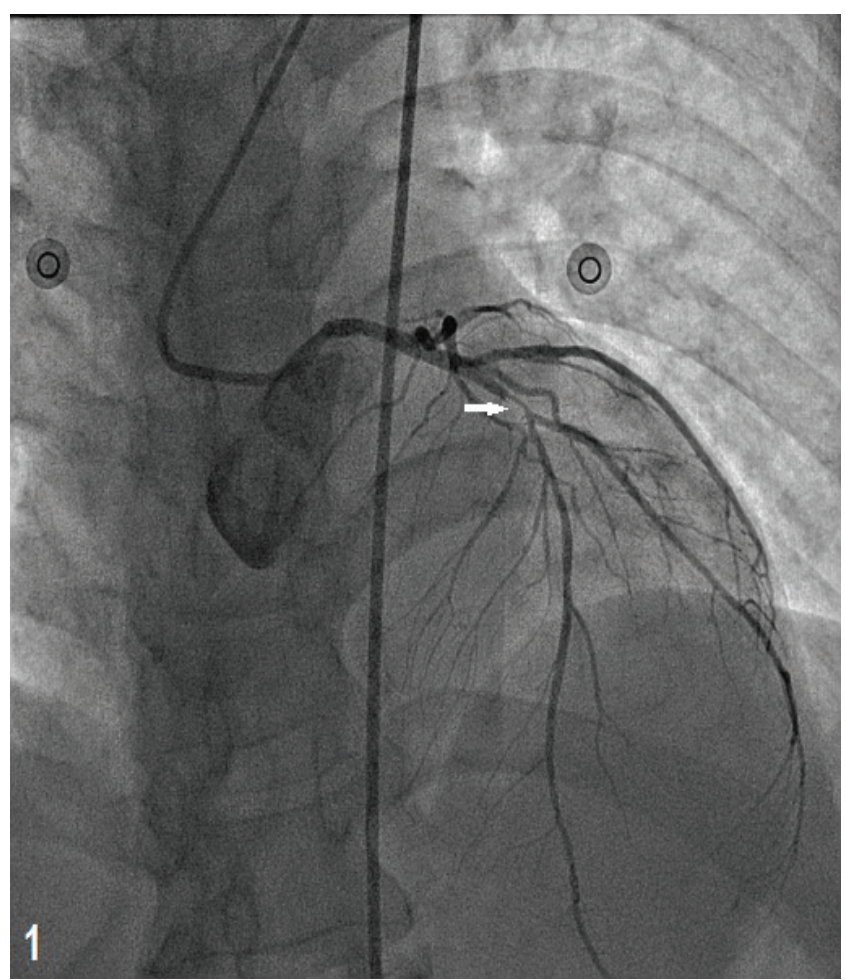

Figure 1: Selective coronary angiography of the left coronary artery using 6 French diagnostic catheter revealed significant obstructive lesion in the mid left anterior descending artery (arrow)

Left circumflex artery and right coronary artery had no significant obstructive lesions. In view of the angiographic findings, $\mathrm{PCl}$ to the LAD was considered. Diagnostic catheter was replaced with a guiding catheter. Selective cannulation of the LAD could not be achieved with either a $6 \mathrm{~F}$ Extra Back-Up guiding catheter (Medtronic) with 3.5 or $4.0 \mathrm{~cm}$ curve. Judkins left 3.5 as well as $4.0 \mathrm{~cm}$ curve guiding catheters were also tried, but the selective cannulation was not successful. Since the patient had severe ongoing chest pain, and also the canulation of the left coronary artery with the guiding catheter was difficult and taking more time, we used the same 6 F Judkins left $3.5 \mathrm{~cm}$ curve diagnostic catheter which was used for the diagnostic coronary angiography. Left coronary artery was engaged with no difficulty. A BMW guidewire (Abbott vascular) was used to cross the lesion. Lesion was predilated with a Sprinter compliant balloon (Medtronic) measuring $2 \times 10 \mathrm{~mm}$ at $12 \mathrm{Atm}$ (Figure 2), followed by the deployment of a cobalt chromium bare metal stent (Presillion Plus, Cordis) measuring $2.75 \times 20$ $\mathrm{mm}$ at $16 \mathrm{Atm}$.

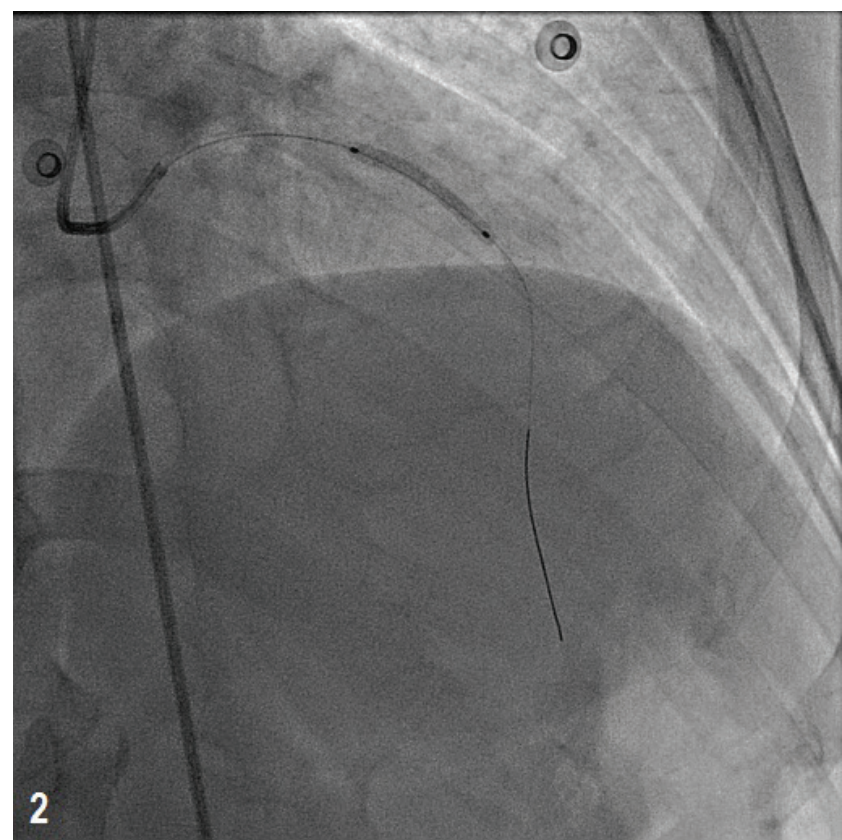

Figure 2: Stent implantation at the mid left anterior descending artery. Same 6 French diagnostic catheter was used for the left coronary artery cannulation

The final result was satisfactory with TIMI III distal flow without residual stenosis or dissection (Figure 3). 10,000 IU unfractionated heparin was given during the procedure. Procedure was uneventful and the patient was transferred to the coronary care unit. Three days later patient was discharged home free of angina.

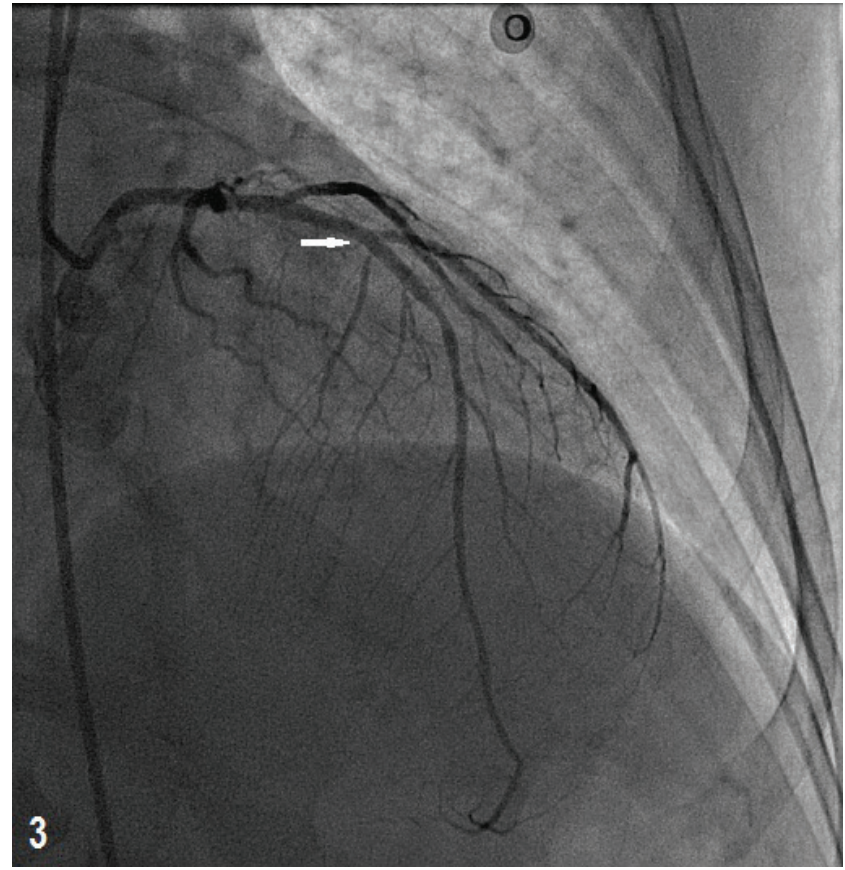

Figure 3: Final result after stenting showed good distal flow without residual stenosis (arrow) 


\section{DISCUSSION}

In 1977, Andreas Gruentzig 5 had performed the first percutaneous transluminal coronary angioplasty in human, since then $\mathrm{PCl}$ has become a mainstay in the treatment of coronary artery disease. In the early years of $\mathrm{PCl}, 9-10 \mathrm{~F}$ guiding catheters were used, whereas $6 \mathrm{~F}$ guiding catheters have now become established as the standard for most cases. ${ }^{6}$ Because of the difference in wall structures of the guiding catheters as compared to the diagnostic catheters and a shorter tip and lack of tapering tip of the guiding catheter, adequate cannulation of the coronary ostium may sometimes be more difficult with guiding catheter. In the era of primary $\mathrm{PCl}$ where time is crucial to salvage the myocardium, difficult coronary ostial cannulation and poor back-up support may be time consuming. Because of its flexibility, a diagnostic catheter can sometimes provide more adequate cannulation as well as greater support during $\mathrm{PCl}$ than a guiding catheter and, thus, can be helpful for successful $\mathrm{PCl}$ within a short period of time.

Resar et al ${ }^{4}$ reported the use of $6 \mathrm{~F}$ diagnostic catheters for balloon angioplasty on 84 lesions in 70 patients with success in $82.9 \%$ of patients. Tzifos et al ${ }^{2}$ reported two cases using $6 \mathrm{~F} \mathrm{di-}$ agnostic catheters to perform $\mathrm{PCl}$. Then Keller et al ${ }^{1}$ reported the feasibility of the $\mathrm{PCl}$ through $6 \mathrm{~F}$ diagnostic catheters in patients with acute coronary syndromes. Those studies have

\section{REFERENCES}

1. Keller PF, Gosselin G, Gregoire J, et al. Feasibility of the $\mathrm{PCl}$ through $6 \mathrm{~F}$ diagnostic catheters. Cathet Cardiovasc Intervent 2007;69:410-5.

2. Tzifos V, Moses J, Oetgen $\mathrm{M}$, et al. Coronary stenting using a $6 \mathrm{~F}$ diagnostic catheter: a report of two cases. Int J Cardiovasc Intervent 2000;3:41-5.

3. Chen YQ, Hou L, Wei YD, et al. Feasibility of using $6 \mathrm{~F}$ angiographic catheters for primary percutaneous coronary intervention in patients demonstrated the feasibility of $\mathrm{PCl}$ via diagnostic catheters.

Though guiding catheter provides adequate arterial blood flow and good visualization of the artery during the $\mathrm{PCl}$ due to its larger lumen diameter as compared to the same size diagnostic catheter, in this case we had accurately positioned and deployed the stent with adequate vessel visualization at all times during the procedure. Back-up support was good with easy positioning of the stent across the stenotic lesion.

\section{CONCLUSION}

Using the same diagnostic catheter used for coronary angiography to perform coronary stenting is useful for patients who have no medical insurance especially in the developing countries like Nepal, as guiding catheters are quite expensive relative to diagnostic catheters. In this case, we successfully used $6 \mathrm{~F}$ diagnostic coronary catheter for both the diagnostic angiography and the subsequent stenting in the mid LAD with good results. Diagnostic $6 \mathrm{~F}$ catheters can easily be used for $\mathrm{PCl}$ in cases where there is difficulty in coronary ostial cannulation and poor back-up support with guiding catheter to save the significant resources as well as the reperfusion time.

\section{Funding: None}

\section{Conflicts of interest: None declared}

with acute myocardial infarction. Chin Med J (Engl) 2010;123:1345-6

4. Resar JR, Prewitt KC, Wolff MR, et al. Percutaneous transluminal coronary angioplasty through $6 \mathrm{~F}$ diagnostic catheters. Am Heart J 1993;125:1591-6.

5. Gruentzig AR. Transluminal dilatation of coronary artery stenosis. Lancet 1978;1:263.

6. Takeshita S, Tanaka S, Saito S. Coronary intervention with 4-French catheters. Cathet Cardiovasc Intervent 2010;75:735-9. 